\title{
EVOLUÇÃO BIBLIOMÉTRICA: UMA ANÁLISE DOS TRABALHOS PUBLICADOS NOS EVENTOS DA ANPAD
}

BIBLIOMETRIC EVOLUTION: AN ANALYSIS OF WORKS PUBLISHED IN ANPAD EVENTS

\author{
Mauri Leodir Löbler', Eliete dos Reis Lehnhart², \\ Rafaela Dutra Tagliapietra ${ }^{3}$, Julia Tontin ${ }^{4}$
}

RECEBIDO: 10/10/2019 | ACEITO: 07/11/2019

DOI: $10.5902 / 2317175840482$

\section{RESUMO}

Esta pesquisa investigou a produção de estudos bibliométricos publicados nos anais de todos os eventos da Associação Nacional de Pós-Graduação e Pesquisa em Administração (AnPAD), no período compreendido entre 2003 e 2019, os quais totalizaram 141 trabalhos examinados. Os resultados indicaram que, embora tenha ocorrido um crescimento no número de estudos bibliométricos, este não foi linear e há um equilíbrio no número de publicações que utilizou os estratos nacional e internacional como fonte base para os estudos bibliométricos investigados. Destaca-se o Encontro da ANPAD (EnANPAD) como o evento com o maior número de estudos bibliométricos publicados e como fonte de busca de dados para a elaboração dos estudos a Web of Science, os eventos realizados pela AnPAD e a Scopus. Observou-se também que os artigos são escritos na maioria das vezes por 2 ou 3 autores com ênfase para as instituições UNINOVE, USP e UNB. Ainda, os resultados evidenciaram o uso de ferramentas computacionais como por exemplo, Microsoft $\circledast$ Office Excel, SPSS, UCINET® e BibExcel. Sendo assim, a pesquisa possibilitou uma visão ampla da produção acadêmica nos principais eventos da área no Brasil com contribuições para o seu desenvolvimento, além de servir como guia para consulta de trabalhos deste campo específico.

Palavras-chave: Bibliometria; Análise bibliométrica; Bibliométrico; Produção científica.

1 Doutor. Professor Adjunto/Universidade Federal de Santa Maria (UFSM).

2 Doutora em Administração. Universidade Federal de Santa Maria (UFSM), Programa de Pós-Graduação em Administração (PPGA); Linha de Pesquisa Tecnologia da Informação e Decisão; Departamento de Ciências Administrativas.

3 Mestra em Administração. Universidade Federal de Santa Maria (UFSM); Departamento de Ciências Administrativas. Curso de Administração.

4 Mestranda em Administração na Universidade Federal de Santa Maria. Departamento de Ciências Administrativas. 


\section{ABSTRACT}

This research investigated the production of bibliometric studies published in the annals of all events of the National Association of Graduate Studies and Research in Administration (AnPAD), from 2003 to 2019, which totaled 141 papers examined. The results indicated that although there was a growth in the number of bibliometric studies, it was not linear and there is a equilibrium in the number of publications that used the national and international strata as the base source for the bibliometric studies investigated. It is highlighted the EnANPAD as the event with the largest number of published bibliometric studies and as a source of data search for the preparation of the studies Web of Science, AnPAD and SCOPUS. It was also observed that the articles are written mostly by 2 or 3 authors with emphasis to UNINOVE, USP and UNB institutions. Also, the results

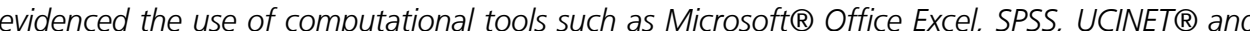
BibExcel. Thus, the research allowed a broad view of academic production in the main events in

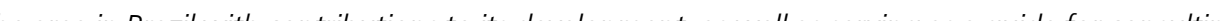
works in this specific field.

Keywords: Bibliometry; Bibliometric analysis; Bibliometric; Scientific production.

\section{Introdução}

A bibliometria pode ser compreendida como a análise quantitativa e estatística utilizada para descrever padrões de publicação dentro de um dado campo da literatura (THANUSKODI, 2010). Logo, os estudos bibliométricos desempenham um papel importante na comunicação das descobertas mais recentes da pesquisa e na publicação de artigos que contenham o desenvolvimento em qualquer campo do conhecimento. Eles refletem vigorosamente tendências emergentes das mais variadas disciplinas (THANUSKODI, 2010) bem como a compreensão sobre o estado da arte, preenchendo determinad lacuna do conhecimento (SANTOS; KOBASHI, 2009). Além disso, no campo de estudos de algumas áreas, como por exemplo, a de ciências administrativas e contabilidade, tal análise permite o entendimento da evolução de dada área do conhecimento em meio às diversas possibilidades que permeiam a produção científica mundial (TREINTA et al., 2014).

Para operacionalização de estudos bibliométricos, de acordo com Araújo et al. (2014) fica à critério do pesquisador justificar as diretrizes de busca, certificando-se que a resposta às hipóteses do estudo esteja adequada a um contexto real. Corroborando essa afirmação, Guedes e Borschiver (2005) acreditam que pesquisa bibliométrica se constitui em uma técnica que permite a constatação de aspectos acerca do conhecimento em investigação, relevantes para o entendimento de determinada temática diante de uma comunidade científica.

Sob essa perspectiva, torna-se viável a compreensão de cada uma das variáveis alvos e que integram o fenômeno ou área de estudo em investigação, "desde a frequência de aparecimento de tema, passando pela localização geográfica das produções e suas respectivas concentrações" (ARAÚJO et al. 2014, p. 97). Assim, de acordo com o levantamento desenvolvido no presen- te estudo, verificou-se que as categorias analíticas nos estudos bibliométricos investigados são as mais diversas, mas possibilitaram a organização e a sistematização das informações coletadas em grupos macro, conforme as categorias fixadas neste estudo. Tais categorias serão detalhadas e aprofundadas na seção de método de pesquisa, exposto a seguir.

Em âmbito nacional, a bibliometria tem sido aplicada nas mais diversas áreas de pesquisa, mas no que diz respeito às ciências sociais aplicadas, como é o caso da administração e contabilidade, o delineamento recebeu enfoques temáticos como, o assédio moral (HEINZMANN; JUNIOR, 2016), a inovação no contexto organizacional (PINTO; MORI; JUNIOR, 2016), a contabilidade pública (FREITAS; SILVA; PRADO, 2017), o poder na referida área (NASCIMENTO et al., 2017), a diversidade humana em estudos de administração (FONSECA; MATITZ, 2018), as políticas públicas na bioeconomia e suas potencialidades (OLIVEIRA et al., 2019) e a governança da educação básica no Brasil (OLIVEIRA, 2019).

Assim, a presente pesquisa visou investigar a produção de estudos bibliométricos na área de ciências da administração e contábeis em âmbito nacional. Com a finalidade de atingir o objetivo fixado, realizou-se uma análise, de cunho quantitativo, dos trabalhos publicados nos eventos da AnPAD, no período compreendido entre 2003 e 2019. O presente artigo está estruturado da seguinte maneira, além da introdução, esta pesquisa contempla um aporte teórico sobre tipos de revisão de literatura e estudos bibliométricos; as diretrizes metodológicas utilizadas para coleta e sistematização dos dados; a discussão e análise dos resultados encontrados, as considerações finais do estudo face às informações geradas e; por fim, as referências adotadas como base para a construção deste artigo.

\section{Referencial teórico}

Esta seção tem por objetivo apresentar uma contextualização acerca do assunto que engloba essa pesquisa visando expor os conceitos teóricos que a fundamentaram. São apresentadas definiç̃̃es sobre bibliometria, a origem do termo, a aplicação do método bibliométrico e a inserção desse tipo de pesquisa na área de administração e contábeis no contexto nacional.

2.1 Estudos bibliométricos

No momento em que se busca reunir informações acerca de determinados assuntos estudados pela ciência, por inúmeras vezes, torna-se um desafio localizar os itens capazes de subsidiar esta tarefa. Os estudos bibliométricos além de visarem preencher essa lacuna (SANTOS; KOBASHI, 2009), também são formas de avaliação do conhecimento científico e de medição de fluxos de informação (VANTI, 2002).

No entender de Pritchard (1969), a bibliometria é a aplicação da matemática e de métodos estatísticos para livros e outros meios de comunicação. Isso 
significa que a bibliometria é uma espécie de técnica de medição pela qual os aspectos interconectados das comunicações escritas podem ser quantificados. O autor ainda considera a bibliometria extensiva a gestão de bibliotecas e instituições envolvidas com o tratamento da informação. Nesse contexto, "os resultados das análises bibliométricas são considerados importantes coadjuvantes da definição de estratégias de gestão de unidades de informação e de bases de dados". Contudo, apesar de autores como Lawani (1981); Sengupta (1992); RoigTierno, Gonzalez-Cruz e Llopis-Martinez (2017) terem atribuído a Pritchard, em 1969, a origem do termo "bibliometrics", segundo Fonseca (1973), foi Paul Otlet quem, no Traité de documentation (1934) usou pela segunda vez a expressão e, ao mesmo tempo cunhou o termo correspondente, em língua francesa, "bibliométrie". Dessa maneira, a partir de um termo anterior "bibliografia estatística" utilizado por Hulme entre 1922 e 1923, originou-se o termo bibliometria.

Para Otlet (1934), autor considerado por historiadores franceses como o criador da bibliometria, foi a partir da bibliologia, ciência que fornece um conjunto sistemático de classificação de dados sobre produção, conservação, circulação e uso de documentos escritos de todos os tipos, que surgiu a ideia de associação dos livros e das medidas, dando origem a bibliometria. Neste contexto, a medida é uma maneira superior de gerar conhecimento. A medição é uma maneira de construir, de forma coordenada, as medidas relativas a livros e a documentos. Tais medidas são relativas aos objetos, aos fenômenos, às relações ou leis. Ela se ocupa especificamente da medida propriamente dita, da parte estatística e da unidade de padronização (OTLET, 1934).

Segundo Thanuskodi (2010), a bibliometria consiste na análise quantitativa e estatística para descrever padrões de publicação dentro de um dado campo da literatura que é utilizada. Sendo assim, pesquisadores usam métodos bibliométricos de avaliação para determinar a influência de um único autor ou para descrever a relação entre dois ou mais autores ou trabalho. Neste sentido, os estudos bibliométricos podem ser usados para análisar padrões regionais de pesquisa, bem como a extensão da cooperação entre grupos e perfis de pesquisa, tendo como principais derivados: contagens de publicações, contagens de citações, análise co-citação, análise co-palavra, mapeamento científico e citações em patentes.

Acrescenta-se a essas considerações o entendimento de Stevens, citado por Sengupta (1992), o qual entende a bibliometria como uma ciência quantitativa e a divide em duas categorias básicas: i) bibliometria descritiva que é usada para produtividade; e ii) bibliometria avaliativa para contar o uso da literatura de um tópico específico, assunto ou disciplina. $O$ autor ainda divide essas duas categorias em diferentes subáreas. A bibliometria descritiva segmentada em: geográfica, período de tempo, e disciplinas, e a bibliometria avaliativa ramificada em: contagem de referência e contagem de citações.

Para elucidar ainda mais, Stevens acrescenta que a bibliometria descritiva inclui o estudo do número de publicações em uma determinada área de pesquisa com a finalidade de comparar a quantidade de pesquisa em diferentes países, a quantidade produzida durante diferentes períodos, ou a quantidade produzida em diferentes subdivisões. Este tipo de estudo é feito por uma contagem de artigos, livros e outros escritos na área de pesquisa ou muitas vezes por uma contagem desses escritos que foram abstraídos em periódicos especializados. Já a bibliometria avaliativa inclui o estudo da literatura utilizada pelos pesquisadores em uma determinada área de pesquisa. Tal estudo é frequentemente feito contando as referências citadas por um grande número de pesquisadores em seus trabalhos (SENGUPTA, 1992).

No entender de Sengupta (1992), o desenvolvimento e crescimento da bibliometria tem sido significativo e se se tornou possível porque motiva muitos pesquisadores de campos distintos do conhecimento a trabalhar em várias facetas da bibliometria. O desenvolvimento mais significativo da bibliometria provavelmente está nas leis fundamentais, a saber: Lei de Lotka (Lei do Inverso Quadrado ou Produtividade Científica), Lei Zipf (Lei da Linguística), e Lei de Bradford (Lei de Dispersão). Essas três leis empíricas proporcionaram um tremendo impulso nas atividades de pesquisa da bibliometria. Baseiam-se respectivamente em: número de autores que contribuem em uma disciplina ou outros campos de estudo; classificação de frequência de palavras em um determinado conjunto de documentos; e distribuição de publicações periódicas em uma área definida do conhecimento ou número de trabalhos em um conjunto de periódicos.

2.2 Estudos bibliométricos no contexto naciona

No Brasil, a bibliometria passou a ser utilizada por volta de 1970, devido à implantação do curso de Mestrado em Ciência da Informação no Instituto Brasileiro de Bibliografia (IBBD), posteriormente denominado Instituto Brasileiro de Informação em Ciência e Tecnologia (IBICT), através da disciplina "Processamento de Dados na Documentação" (ALVARADO, 1984). Em 1984, no trabalho intitulado "A Bibliometria no Brasil", Rubén Urbizagástegui Alvarado analisou as publicações no período de 1972 a 1983 e encontrou 78 trabalhos que utilizaram a bibliometria. Neste trabalho, Alvarado constatou que os autores introdutores da bibliometria no Brasil foram Tefko Saracevic, Bert Boyce e Wilfred Lancaster, e que, apesar de apresentar trabalhos em diversas áreas, a maior concentração da produção ocorreu nos cursos de Ciência da Informação do IBICT.

No ano de 2010, Meneghini e Packer publicaram o artigo "The Extent of Multidisciplinary Authorship of Articles on Scientometrics and Bibliometrics in Brazil". Neste trabalho foram analisados artigos publicados no Brasil no período de 1990 a 2006. Foram encontrados 197 artigos e pôde-se verificar que apenas um quarto dos artigos estava relacionado à área ciência da informação, os demais estavam distribuídos nas áreas de humanas/administração, biologia/biomedicina, saúde e ciências exatas. Isto evidencia a forte tendência do crescente uso dos estudos bibliométricos em diversas áreas, inclusive na administração. 
No campo da administração, a produção de artigos bibliométricos se apresenta em ascensão. Dentre os vários estudos com essa particularidade é importante mencionar algumas publicações que foram acontecendo no decorrer dos últimos anos, como por exemplo, em 2013, o artigo de Teixeira et al. "Estudos Bibliométricos em Administração: Discutindo a Transposição de Finalidade". Neste trabalho foram analisados artigos publicados em eventos e revistas de administração no período de 2003 a 2012. O resultado apontou 36 artigos bibliométricos produzidos somente na área de administração, sendo sua predominância no sul e sudeste do país. Desta forma, pode-se observa a importância dos estudos bibliométricos e seu aumento expressivo nos mais variados campos da ciência.

Em 2014, Araújo e colaboradores publicaram um estudo exploratóriobibliométrico tendo por objetivo analisar todos os periódicos da área de Administração, Ciências Contábeis e Turismo. Por meio de uma análise documental realizada nos sites de todas as revistas classificadas de A1 a B5 pelo QUALIS/CAPES (Triênio 2009-2011), os autores concluíram que há despreparo de algumas gestões editoriais no processo de acompanhamento e execução as atividades inerentes ao processo editorial. Há uma concentração de periódicos com maior qualificação nas regiões Sul e Sudeste e há poucos periódicos de alto impacto na perspectiva nacional, bem como a existência de grande parte da produção impressa, limitando o acesso ao conhecimento (ARAÚJO et al., 2014).

No ano de 2017, Nascimento e colegas publicaram um estudo biliométrico cujo o objetivo foi analisar a produção científica brasileira sobre o poder na área de Administração, Administração Pública, Contabilidade e Turismo. Os autores observaram movimentos de ascensão e declínio das publicações no período es tudado (2006 a 2016). Os temas mais abordados foram relações, redes e conhecimento. As pesquisas eram empíricas, de abordagem qualitativa, de natureza descritiva, predominando o procedimento estudo de caso por meio de entrevista e a análise de conteúdo. Ainda constataram que o setor mais estudado são as instituições de ensino superior e o perfil de autoria é de dois autores por artigo, sendo doutores da região Sudeste (NASCIMENTO et al., 2017).

Este cenário serve para embasar as suposições de Roig-Tierno, Gonzalez-Cruz e Llopis-Martinez (2017) ao afirmarem que o campo da bibliometria cresceu exponencialmente desde o advento da Internet, o que facilitou a comunicação entre pesquisadores de todo o mundo e permitiu acesso às contribuições em uma determinada área. Cabe lembrar que os trabalhos acima mencionados são alguns exemplos, dentre vários estudos bibliométricos que têm sido publicados na área de Administração, Contabilidade e afins no Brasil e, de modo algum esgotam o leque de pesquisas bibliométricas realizadas nessa área de estudos.

De acordo com Jonkers e Derrick (2012) quando usada de maneira apropriada a bibliometria oferece ferramentas úteis para a tomada de decisão sobre a pesquisa, além de ajudar a definir prioridades de investigação, mapear o desenvolvimento da ciência e propiciar pesquisa de excelência. A informação científica pode servir funções distintas: a de educar, fornecendo análises e explicações sobre existente conhecimento; para debater o conhecimento; e criar novos conhecimentos. Em relação ao conhecimento bibliométrico, pode-se dizer que a legitimidade dos estudos e seu uso continuado dependem da entrada e participação de cientistas dos mais diversos campos da ciência (JONKERS; DERICK, 2012).

\section{Método de pesquisa}

A fim de investigar a produção de estudos bibliométricos na área de ciências da administração, contábeis e afins em âmbito nacional, realizou-se um trabalho de natureza quantitativa e descritiva. Para tanto, por meio de um estudo bibliométrico, analisaram-se 141 artigos publicados no período de 2003 a 2019, nos Anais dos eventos da AnPAD. A coleta foi feita mediante pesquisa eletrônica na base de dados de todos os eventos da AnPAD, tendo como critério de busca, os artigos que contivessem no título o termo "bibliom". Com relação ao período investigado, o ano de 2003 é a referência inicial pela disponibilidade dos trabalhos publicados no site da AnPAD a partir desse período e o ano de 2019 é a referência final considerando os eventos que já haviam acontecido até o mês de junho. Ademais, considerou-se o período de busca de dezesseis anos, suficiente para que fosse compreendida a evolução dos estudos bibliométricos na área investigada.

A motivação pela escolha da base de dados dos eventos da AnPAD se deve ao fato da relevância e reconhecimento nacional de pesquisadores que publicam seus trabalhos nesses eventos, fato que torna bom indicador do desenvolvimento da produção científica nessa área de conhecimento no Brasil (ALBUQUERQUE; ANDRADE; JOIA, 2010). A partir das afirmações, constata-se que os artigos encontrados em seus anais são fonte segura de consulta bibliométrica. Cumpre ser ressaltado que a escolha de eventos nacionais tem a intenção de analisar a produção científica brasileira voltada para a divulgação de trabalhos em Administração, Contábeis e afins e sua evolução.

Após o resultado das buscas, que totalizou em 144 trabalhos, o procedimento para inclusão ou exclusão do artigo no escopo da pesquisa consistiu na análise da integralidade do próprio artigo, ou seja, foram excluídos os trabalhos que não possuíam texto completo disponível ou que apresentavam somente o resumo. Dessa maneira, foram retirados da amostra 3 artigos, cujas as informações não eram suficientes para mantê-los no propósito da pesquisa. Conforme Café e Bräscher (2008), para que a seleção inclua documentos científicos com propriedades similares, é essencial a padronização da descrição física e de conteúdo desses documentos. Posteriormente, os 141 artigos selecionados, foram separados por ano e por evento onde foram publicados, antes da realização de leitura dos mesmos. 
O próximo procedimento consistiu na leitura do resumo dos estudos, bem como no preenchimento de uma planilha no Microsoft Windows Exce/® em relação as métricas de publicação de interesse dessa pesquisa. Em quase totalidade dos casos, houve a leitura integral da seção de metodologia das pesquisas, a fim de identificar o período selecionado para a análise bibliométrica dos artigos, a abrangência, o título da fonte, a base de dados utilizada, a ferramenta de apoio para as análises, dentre outros aspectos.

Posteriormente à leitura e análise dos artigos, a apresentação dos resultados desse estudo foi organizada de acordo com: i) o período analisado; ii) evento e a divisão temática da AnPAD; iii) a autoria e a filiação institucional bem como a quantidade de autores; iv) a abrangência, sendo executado em âmbito nacional, internacional e ambos os âmbitos de pesquisa; v) o título da fonte, se periódico ou anais de eventos; com a extensão do período analisado pelos estudos bibliométricos; vi) a base de dados utilizada; vii) a adoção de softwares e ferramentas para operacionalização e sistematização dos dados obtidos pelos estudos; e, por fim, viii) a ocorrência de palavras-chaves dos estudos. A Figura 1 ilustra essas categorias de análise.

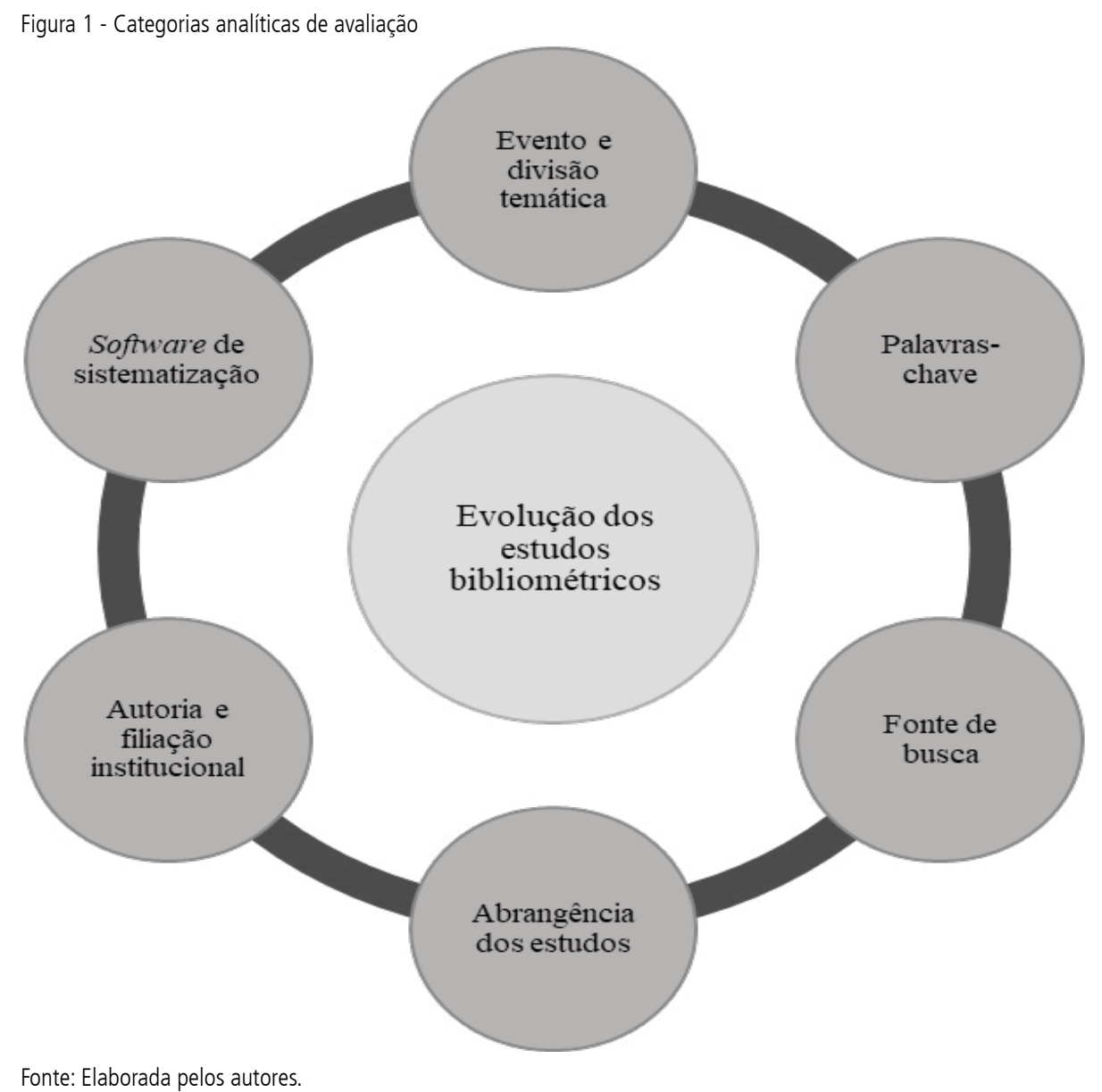

Quanto à análise dos dados, como auxílio do Microsoft Windows Exce/® e do Word Cloud Generator, plugin para o navegador Chrome para criar nuvem de palavras, ela foi realizada a partir de frequências com base nas categorias analíticas, apresentadas em tabelas e gráficos e formação de nuvem de palavra. Suas discussões ocorreram descritivamente tendo em vista o alcance do objetivo proposto neste estudo.

\section{Apresentação e discussão dos resultados}

Os elementos considerados, a partir dos artigos incluídos para análise nessa pesquisa, são apresentados nas próximas subseções e se referem suas distribuições de acordo com as categorias analíticas mencionadas na Figura 1. Como pode-se observar pelos dados da Figura 2, de 2003 até 2008 houve um crescimento tênue nas publicações de estudos bibliométricos. No entanto, no ano de 2009 há uma queda no número de publicações e consequente uma retomada no crescimento a partir de 2010, seguindo até 2013, quando em 2014 novamente observa-se uma oscilação decrescente nas pesquisas bibliométricas. Embora em 2015 aconteça um crescimento no número de publicações, esse comportamento não se mantém constante, uma vez que a partir de 2016 inicia-se uma diminuição no número desse tipo de estudo, a qual vai até meados de 2019, limite do corte transversal dos artigos investigados nesta pesquisa.

Dessa maneira, destaca-se que, até o ano de 2011, houve um espaço temporal com poucas publicações, e, somente a partir do ano de 2012, os estudos bibliométricos começam a mostrar-se em maior número nos anais da AnPAD. Esses resultados levam a acreditar que esses tipos de estudos têm se apresentado como importantes fontes de informação sobre as tendências de pesquisa em distintos campos de análise.

Figura 2 - Distribuição dos artigos no período analisade

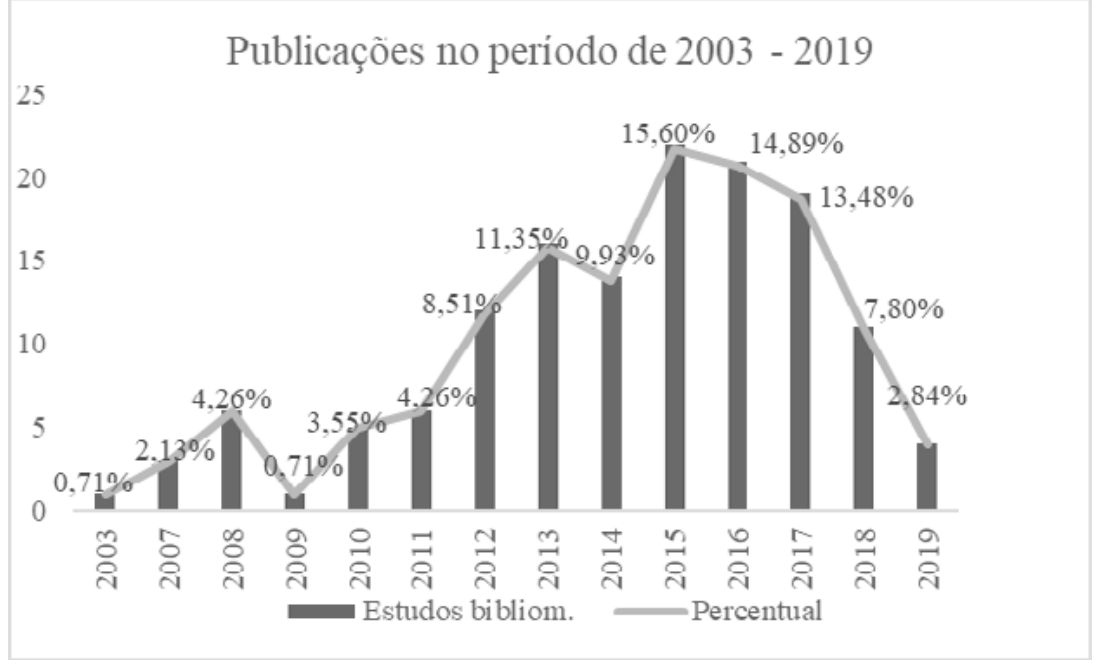

Fonte: Dados da pesquisa. 
Ao analisar o número dos estudos bibliométricos de acordo com os eventos da AnPAD, por meio dos dados da Tabela 1 é possível observar que 0 EnANPAD lidera o ranking com 99 (70,21\%) artigos publicados entre os eventos temáticos. Esse resultado é consoante aos achados de Teixeira, Iwamoto Medeiros (2013), uma vez que, em estudo realizado pelas autoras, foi identificado que a grande maioria das publicações também ocorreu no EnANPAD.

Em segundo lugar e com o mesmo número de publicações (7 - 4,96\% cada) estão os eventos Simpósio e Encontro de Estudos em Estratégia (3Es). Em contrapartida, com o menor número de publicações $(4-2,83 \%$ cada) estão os eventos Encontro de Administração da Informação (EnADI), Encontro de Ensino e Pesquisa em Administração e Contabilidade (EnEPQ) e Encontro de Estudos Organizacionais da ANPAD (EnEO). Esse comportamento pode estar associado ao fato do EnANPAD ser considerado o maior evento da AnPAD a qual desenvolve um consistente trabaIho na promoção do ensino, da pesquisa e na produção de conhecimento dentro do campo das ciências administrativas, contábeis e afins no Brasil (ANPAD, 2019).

No que se refere as divisões temáticas dos eventos, pode-se perceber que liderando e com igual número de publicações (19 cada), estão as divisões EPQ e ESO do EnANPAD. Por outro lado, a divisão que teve o menor número (1) de estudos bibliométricos publicados foi a APS, também do EnANPAD. Ademais, nas outras divisões, o número de estudos oscila, entre 7 e 4, sendo o Simpósio e o $3 E s$ os líderes com 7 publicações e as divisões dos eventos EnADI, EnEPQ e EnEO com 4 cada uma.

Tabela 1 - Distribuição dos estudos de acordo com o Evento

\begin{tabular}{|c|c|c|c|}
\hline Evento & Divisão & Frequência & Percentual \\
\hline \multirow{11}{*}{ EnANPAD } & Ensino e Pesquisa em Administração e Contabilidade (EPQ) & 19 & $13,48 \%$ \\
\hline & $\begin{array}{l}\text { Estratégia em Organizações (ESO) } \\
\end{array}$ & 19 & $13,48 \%$ \\
\hline & Gestão de Pessoas e Relações de Trabalho (GPR) & 11 & $7,80 \%$ \\
\hline & Gestão de Ciência, Tecnologia e Inovação (GCT) & 11 & $7,80 \%$ \\
\hline & Gestão de Operaçõos e Logística (GOL) & 10 & $7,09 \%$ \\
\hline & Administração da Informação (ADI) & 7 & $4,96 \%$ \\
\hline & Administração Pública (APB) & 7 & $4,96 \%$ \\
\hline & Marketing (MKT) & 6 & $4,26 \%$ \\
\hline & Estudos Organizacionais (EOR) & 5 & $3,55 \%$ \\
\hline & Finanças (FIN) & 3 & $2,13 \%$ \\
\hline & Administração Pública e Gestão Social (APS) & 1 & $0,71 \%$ \\
\hline SIMPÓSIO & SIMPÓSIO & 7 & $4,96 \%$ \\
\hline 3ES & Encontro de Estudos em Estratégia (3Es) & 7 & $4,96 \%$ \\
\hline EnGPR & Encontro de Gestão de Pessoas e Relaçốes de Trabalho (EnGPR) & 6 & $4,26 \%$ \\
\hline EnAPG & Encontro de Administração Pública da ANPAD (EnAPG) & 5 & $3,55 \%$ \\
\hline EMA & Encontro de Marketing da ANPAD (EMA) & 5 & $3,55 \%$ \\
\hline EnADI & Encontro de Administração da Informação (EnADI) & 4 & $2,84 \%$ \\
\hline EnEPQ & Encontro de Ensino e Pesquisa em Administração e Contabilidade (EnEPQ) & 4 & $2,84 \%$ \\
\hline EnEO & Encontro de Estudos Organizacionais da ANPAD (EnEO) & 4 & $2,84 \%$ \\
\hline Total & & 141 & $100,00 \%$ \\
\hline
\end{tabular}

RSh REVISTA SOCIAIS \& HUMANAS - VOL. 32 / No 3 - 2019
A maior frequência de publicações observada nas divisões do EPQ e ESO provavelmente dá-se pelas características das temáticas, ou seja, os estudos bibliométricos podem ser considerados um meio para divulgação das estratégias de pesquisa nas áreas de administração, contabilidade e afins, nos mais variados assuntos dessa área de estudo. Ademais, a ampla possibilidade de pesquisas envolvendo a questão de estratégia em organizações pode ter contribuído para esse resultado.

A fim de verificar a abrangência dos artigos selecionados para composição dos estudos bibliométricos investigados nessa pesquisa, conforme os dados da Tabela 2, pode-se perceber um equilíbrio entre os estratos nacional e internacional, ou seja, dos 141 estudos analisados $56(39,72 \%)$ tomaram como base estudos nacionais e $52(36,87 \%)$ estudos internacionais. Quanto a abrangência em ambos os contextos, $33(23,41 \%)$ dos estudos recorreram a pesquisas publicadas no Brasil e no exterior. Esses resultados demonstram uma congruência na busca por pesquisas significativas, considerando também o contexto externo. Talvez esses achados sinalizem que, embora exista forte interesse na pesquisa internacional, ainda há espaço para maior busca e novas perspectivas dos assuntos que estão sendo desenvolvidos no contexto internacional.

\begin{tabular}{ccccccc}
\multicolumn{7}{l}{ Tabela 2 - Distribuição dos estudos de acordo com a abrangência } \\
\hline Ano & Nacional & Perc. & Internacional & Perc. & $\begin{array}{c}\text { Nacionall } \\
\text { Internacional }\end{array}$ & Perc. \\
\hline 2003 & 1 & $1,79 \%$ & 0 & $0,00 \%$ & 0 & $0,00 \%$ \\
\hline 2007 & 2 & $3,57 \%$ & 0 & $0,00 \%$ & 1 & $3,03 \%$ \\
\hline 2008 & 4 & $7,14 \%$ & 1 & $1,96 \%$ & 1 & $3,03 \%$ \\
\hline 2009 & 1 & $1,79 \%$ & 0 & $0,00 \%$ & 0 & $0,00 \%$ \\
\hline 2010 & 2 & $3,57 \%$ & 2 & $3,92 \%$ & 1 & $3,03 \%$ \\
\hline 2011 & 4 & $7,14 \%$ & 2 & $3,92 \%$ & 0 & $0,00 \%$ \\
\hline 2012 & 6 & $10,71 \%$ & 3 & $5,88 \%$ & 3 & $9,09 \%$ \\
\hline 2013 & 5 & $8,93 \%$ & 7 & $13,73 \%$ & 4 & $12,12 \%$ \\
\hline 2014 & 7 & $12,50 \%$ & 4 & $7,84 \%$ & 3 & $9,09 \%$ \\
\hline 2015 & 9 & $16,07 \%$ & 9 & $15,69 \%$ & 4 & $12,12 \%$ \\
\hline 2016 & 3 & $5,36 \%$ & 12 & $23,53 \%$ & 6 & $18,18 \%$ \\
\hline 2017 & 9 & $16,07 \%$ & 7 & $13,73 \%$ & 3 & $9,09 \%$ \\
\hline 2018 & 2 & $3,57 \%$ & 5 & $9,80 \%$ & 4 & $12,12 \%$ \\
\hline 2019 & 1 & $1,79 \%$ & 0 & $0,00 \%$ & 3 & $9,09 \%$ \\
\hline Total & $\mathbf{5 6}$ & $\mathbf{1 0 0 , 0 0 \%}$ & $\mathbf{5 2}$ & $\mathbf{1 0 0 , 0 0 \%}$ & $\mathbf{3 3}$ & $\mathbf{1 0 0 , 0 0 \%}$ \\
\hline Fonte: Dados da pesquisa. & & & & &
\end{tabular}

Com o propósito de verificar a incidência de co-autores nos estudos pesquisados, realizou-se a contagem do número de autores por artigo. Os dados da Tabela 3 apontam que os estudos foram realizados por no máximo 6 pesquisadores, sendo que o número predominante foi de 3 autores por artigo, ou seja, $35,46 \%$, seguidos de $24,11 \%$ por 2 autores e $19,86 \%$ com 4 autores. Esse resultado se assemelha aos achados de outros estudos que também investigaram o número de pesquisadores por artigo publicado, como por exemplo Nas- 
cimento et al. (2017), Splitter, da Rosa e Borba (2012), Chiareto et al., (2015). Embora em contextos temáticos específicos, o número de autores mais frequentes nos estudos bibliométricos desses autores também oscilou entre 2 e 3 .

Tabela 3 - Distribuição dos estudos de acordo com a quantidade de autores

\begin{tabular}{lll}
\hline Quantidade de autores & Frequência & Percentual \\
\hline 1 autor & 10 & $7,09 \%$ \\
\hline 2 autores & 34 & $24,11 \%$ \\
\hline 3 autores & 50 & $35,46 \%$ \\
\hline 4 autores & 28 & $19,86 \%$ \\
\hline 5 autores & 13 & $9,22 \%$ \\
\hline 6 autores & 6 & $4,26 \%$ \\
\hline Total & $\mathbf{1 4 1}$ & $\mathbf{1 0 0 , 0 0 \%}$
\end{tabular}

Total

$100,00 \%$

De acordo com a análise desenvolvida em cada um dos artigos bibliométricos pertencentes à amostra, verificou-se que a categoria de análise relacionada à autoria foi fortemente destacada nos resultados dos estudos. Para a definição da relação de autoria, levou-se em consideração o nome de todos os autores dos artigos da amostra pesquisada. Tendo em vista sua relevância, a Tabela 4 retrata a distribuição dos trabalhos em função dos pesquisadores envolvidos em sua elaboração.

Tabela 4 - Distribuição dos estudos de acordo com a autoria

\begin{tabular}{lll}
\hline Autoria & Frequência & Percentual \\
\hline Manuel Portugal Ferreira & 6 & $9,37 \%$ \\
\hline Fernando Antonio Ribeiro Serra & 3 & $4,68 \%$ \\
\hline Nuno Rosa Reis & 3 & $4,68 \%$ \\
\hline Ana Paula Mussi Szabo Cherobim & 2 & $3,12 \%$ \\
\hline André Andrade Longaray & 2 & $3,12 \%$ \\
\hline Angelo Palmisano & 2 & $3,12 \%$ \\
\hline Augusto Cézzar de Aquino Cabral & 2 & $3,12 \%$ \\
\hline Damiana Machado de Almeida & 2 & $3,12 \%$ \\
\hline Eliete dos Reis Lehnhart & 2 & $3,12 \%$ \\
\hline Evandro Luiz Lopes & 2 & $3,12 \%$ \\
\hline Fernando Ribeiro Serra & 2 & $3,12 \%$ \\
\hline Gisela Demo & 2 & $3,12 \%$ \\
\hline Henrique César Melo Ribeiro & 2 & $3,12 \%$ \\
\hline Jonathan Saidelles Corrêa & 2 & $3,12 \%$ \\
\hline Júlio César Bastos de Figueiredo & 2 & $3,12 \%$ \\
\hline Kelly Carvalho Vieira & 2 & $3,12 \%$ \\
\hline Lucas Veiga Avila & 2 & $3,12 \%$ \\
\hline Lú́cia dos Santos Albanio & 2 & $3,12 \%$ \\
\hline Luis Felipe Dias Lopes & 2 & $3,12 \%$ \\
\hline
\end{tabular}

RSh REVISTA SOCIAIS \& HUMANAS - VOL. 32 / No 3 - 2019

\begin{tabular}{lll}
\hline Luiz Antonio de Camargo Guerrazzi & 2 & $3,12 \%$ \\
\hline Maria Emilia Camargo & 2 & $3,12 \%$ \\
\hline Mauri Leodir Löbler & 2 & $3,12 \%$ \\
\hline Natasha Fogaça & 2 & $3,12 \%$ \\
\hline Rodrigo Antonio Martins & 2 & $3,12 \%$ \\
\hline Rogério Ruas Machado & 2 & $3,12 \%$ \\
\hline Sandra Maria dos Santos & 2 & $3,12 \%$ \\
\hline Silvana Anita Walter & 2 & $3,12 \%$ \\
\hline Saulo Fabiano Amâncio Vieira & 2 & $3,12 \%$ \\
\hline Tatiana Marceda Bach & 2 & $3,12 \%$ \\
\hline Total & 64 & $\mathbf{1 0 0 , 0 0} \%$ \\
\hline Fonte: Dados da pesquisa & &
\end{tabular}

Total

Para composição da tabela, a frequência de autoria dos pesquisadores sofreu um recorte de, no mínimo, duas publicações entre os estudos analisados. Desse modo, a partir da análise dos dados contidos na tabela, verifica-se que o pesquisador Manuel Portugal Ferreira desenvolveu seis estudos bibliométricos no período analisado. Atualmente, de acordo com informações retiradas do Currículo Lattes, o referido autor atua como professor e investigador nas áreas de estratégia e gestão internacional, na Universidade Nove de Julho, no Programa de Mestrado e Doutorado em Administração, São Paulo, Brasil. Além disso, concluiu seus estudos como pós-doutorado na FEA/USP e desenvolveu seu doutorado em Business Administration pela David Eccles School of Business da Universidade de Utah, EUA.

Na sequência, com a publicação de três artigos, aparecem os autores Fernando Antonio Ribeiro Serra, com filiação institucional na Universidade Nove de Julho (UNINOVE), São Paulo, Brasil, e Nuno Rosa Reis, com filiação institucional na Faculdade de Economia da Universidade de Coimbra, Portugal. Ressalta-se que os autores com maior número de estudos bibliométricos publicaram seus estudos em co-autoria. Ademais, verificou-se que grande grupo de estudiosos ficou ranqueado com a mesma representatividade de desenvolvimento de estudos bibliométricos, cada autor com dois títulos publicados durante o espaço de tempo investigado.

Com o intuito de analisar a filiação institucional, a qual a maioria dos pesquisadores está vinculada, procedeu-se à contagem da ocorrência das instituições na amostra de estudos pesquisada. A Tabela 5 apresenta tal achado.

Tabela 5 - Distribuição dos estudos de acordo com a filiação instituciona

\begin{tabular}{lll}
\hline Instituição & Frequência & Percentual \\
\hline Universidade Nove de Julho & 18 & $15,65 \%$ \\
\hline Universidade de São Paulo & 17 & $14,78 \%$ \\
\hline Universidade de Brasilila & 16 & $13,91 \%$ \\
\hline Universidade Federal de Santa Catarina & 14 & $12,17 \%$ \\
\hline Escola de Administraçãao de Empresas de São Paulo & 12 & $10,43 \%$ \\
\hline Universidade Federal do Paraná & 7 & $6,08 \%$ \\
\hline
\end{tabular}

Universidade Federal do Paraná 


\begin{tabular}{lll}
\hline Universidade Federal de Minas Gerais & 6 & $5,21 \%$ \\
\hline Universidade Federal de Santa Maria & 6 & $5,21 \%$ \\
\hline Universidade Federal do Ceará & 6 & $5,21 \%$ \\
\hline Instituto Politécnico de Leiria & 5 & $4,34 \%$ \\
\hline Universidade Federal de Lavras & 4 & $3,47 \%$ \\
\hline Universidade Regional de Blumenau & 4 & $3,47 \%$ \\
\hline Total & $\mathbf{1 1 5}$ & $\mathbf{1 0 0 , 0 0 \%}$ \\
\hline
\end{tabular}

Fonte: Dados da pesquisa.

Com base nos dados da tabela, conclui-se que as instituições com maio frequência em estudos bibliométricos foram, a Universidade Nove de Julho (UNINOVE), representando 15,65\%; a Universidade de São Paulo, representando 14,78\%; a Universidade de Brasília, representando 13,91\%; a Universidade Federal de Santa Catarina, representando 12,17\%; e a Escola de Administração de Empresas de São Paulo, representando 10,43\%. Este resultado pode ser relacionado com a expressividade de autoria identificada na Tabela 4, na qual verificou-se que os três autores com maior número de publicações estão vinculados a instituição com maior número de estudos bibliométricos, a Universidade Nove de Julho (UNINOVE).

No que se refere ao uso de ferramentas de apoio para a análise dos dados e elaboração dos estudos bibliométricos, dos 141 artigos, 56 (39,71\%) não mencionaram o uso de alguma ferramenta. Por meio dos dados da Tabela 6 é possivel observar que a planilha eletrônica Excel foi a mais utilizada, seguida do Statistical Package for the Social Sciences (SPSS). Há também relativa aplicação dos softwares UCINET $\circledast$, BibExcel, EndNote $\circledast$ e VOSviewer. De acordo com Ruas e Pereira (2014), esses softwares são úteis para busca, extração e visualização de dados dos repositórios de publicações científicas e para construção de indicadores de produtividade científica e tecnológica.

Tabela 6 - Distribuição dos estudos de acordo com os softwares utilizados

\begin{tabular}{ccc}
\hline Ferramenta & Frequência & Percentual \\
\hline Microsoft $₫$ Office Excel & 33 & $23,40 \%$ \\
\hline SPSS & 21 & $14,89 \%$ \\
\hline UCINET $\Theta$ & 18 & $12,77 \%$ \\
\hline BibExcel & 13 & $9,22 \%$ \\
\hline EndNote ${ }^{2}$ & 8 & $5,67 \%$ \\
\hline VOSviewer & 7 & $4,96 \%$ \\
\hline Wordle & 6 & $4,26 \%$ \\
\hline Publish or Perish & 4 & $2,84 \%$ \\
\hline CiteSpace & 3 & $2,13 \%$ \\
\hline ProKnow-C & 3 & $2,13 \%$ \\
\hline Outras & 31 & $21,99 \%$ \\
\hline Total & $\mathbf{1 4 7}$ & $\mathbf{1 0 4 , 2 6 \%}$ \\
\hline
\end{tabular}

RSh REVISTA SOCIAIS \& HUMANAS - VOL. 32 / ํo 3 - 2019
Ao que se refere à categoria "outras", destacada na Tabela 6, esta diz respeito as ferramentas utilizadas para a análise dos dados nos artigos estudados que obtiveram frequência igual ou menor que 2. Estas ferramentas foram: HistCite; Mendeley; NetDraw ${ }^{\circledR}$; Pajek; R; Sitkis; Bibliometrix; CitNetExplorer; Dataming; Draw; Gephi; Harzing's; Index Ordinatio; Iramuteq; Lexica $\Theta_{i}$ ManyEyes; Mplus; Nvivo10; Sphinix; STATA; TagCrowd; Textming; Tagxedo; Wordclouds.com; Wordcounter360

Dentre os 141 estudos da amostra investigada, verificou-se a categoria de análise relacionada à fonte de busca adotada pelos artigos. A Tabela 7, exposta a seguir, detalha a frequência de utilização no que tange à fonte de busca disponível para o desenvolvimento de estudos bibliométricos. Ressaltase que, para composição da tabela, a ocorrência de utilização das fontes sofreu um recorte de, no mínimo, três citações entre os estudos analisados. Além disso, destaca-se que alguns estudos utilizaram mais de uma fonte de busca para coleta de dados, ocasionando em uma frequência de 160 resultados.

\begin{tabular}{|c|c|c|}
\hline Fonte de busca & Frequência & Percentual \\
\hline Web of Science & 48 & $30,00 \%$ \\
\hline Eventos da AnPAD & 28 & $17,50 \%$ \\
\hline Scopus & 23 & $14,38 \%$ \\
\hline Portal Capes & 16 & $10,00 \%$ \\
\hline SPELL & 16 & $10,00 \%$ \\
\hline SCiELO & 9 & $5,62 \%$ \\
\hline EBSCO & 7 & $4,37 \%$ \\
\hline Google Scholar & 4 & $2,50 \%$ \\
\hline Brazilian Administration Review & 3 & $1,87 \%$ \\
\hline Emerald Publishing & 3 & $1,87 \%$ \\
\hline Revista de Administraçãoo Contemporânea & 3 & $1,87 \%$ \\
\hline Total & 160 & $100 \%$ \\
\hline
\end{tabular}

Fonte: Dados da pesquisa.

No que se refere às fontes de busca utilizadas pelos estudos, constatouse que a maioria dos artigos realizou o levantamento por meio da Web of Science, correspondendo a 30,00\% do total da amostra investigada. Esse resultado deve-se ao fato dessa base de dados atender aos critérios de relevância e reconhecimento nacional e internacional de pesquisadores, bem como pertencer a plataforma do Portal Capes e possuir um vasto acervo de publicações (BOTELHO et al., 2011).

m seguida, verificou-se que os anais da AnPAD foi a segunda fonte de busca mais utilizada pelos artigos bibliométricos. Em âmbito nacional, os trabaIhos publicados nos eventos promovidos pela AnPAD constituem bom indicador a respeito da evolução da produção científica na área de ciências administrativas, contábeis e afins (HOPPEN; MEIRELLES, 2005, SORDI et al., 2013). Em função 
disso, justifica-se como consistente fonte de busca para o desenvolvimento de estudos bibliométricos (ALBUQUERQUE; ANDRADE; JOIA, 2010), corroborando a utilização da referida fonte, representando $17,50 \%$ dos estudos analisados.

Na sequência, identificou-se a fonte de levantamento de dados Scopus com ocorrência de $14,38 \%$ dentre os estudos investigados. Adicionalmente, as fontes de busca Portal Capes e Spel/ representaram 10,00\% de ocorrência para o levantamento de dados em estudos bibliométricos. Vale destacar que o Portal Capes, configura-se como uma biblioteca virtual que disponibiliza a produção científica internacional, por meio de um acervo de, aproximadamente, 45 mil títulos com texto completo, 130 bases referenciais, 12 bases dedicadas exclusivamente a patentes, além de livros, enciclopédias e obras de referência, normas técnicas, estatísticas e conteúdo audiovisual (PORTAL CAPES, 2019).

Por fim, ainda no que diz respeito à fonte de busca escolhida pelos pesquisadores, verificou-se com menor expressividade de utilização as seguintes fontes e suas representatividades, SCiELO (5,62\%), EBSCO (4,37\%), Google Scholar (2,50\%), Brazilian Administration Review (1,87\%), Emerald Publishing (1,87\%), Revista de Administração Contemporânea RAC (1,87\%), respectivamente.

Adicionalmente, a verificação de frequência de palavras-chave foi uma das categorias de análise investigada no presente artigo. Para tanto, procedeu-se a contagem da ocorrência das palavras-chave expressas nos estudos bibliométricos, totalizando 413 palavras.

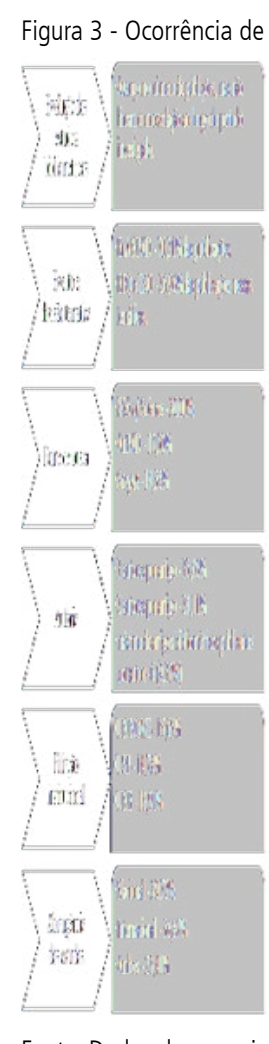

Fonte: Dados da pesquisa.

$$
\text { alavras-chave nos estudos }
$$
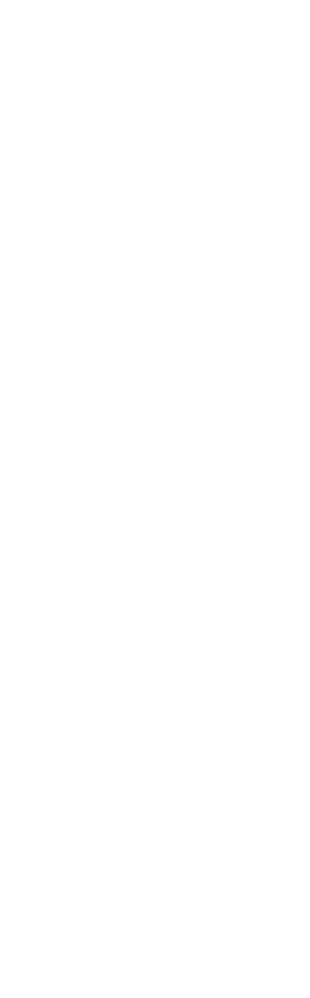

A Figura 3, ilustra as palavras com maior ocorrência dentre os estudos analisados. Inicialmente, para a operacionalização da nuvem de palavras, utilizou-se o site Word Clouds, bem como os 94 estudos que continham palavras-chave fixadas no documento, correspondendo a $66,66 \%$ da amostra investigada. Salientase que, 47 estudos não apresentaram o referido elemento, pois acredita-se que os eventos da AnPAD passaram a tornar tal aspecto exigência nas instruções de formatação dos artigos apenas nos últimos anos, posto que a amostra analisada contempla todos os estudos bibliométricos publicados a partir de 2003.

Conforme constatou-se neste levantamento, grande parte dos artigos que inclui as palavras-chaves em seus achados adota sites e softwares para no formato do Word Clouds para apresentação do resultado. No que diz respeito às terminologias ilustradas, conclui-se que as palavras-chave de maior ocorrência foram "bibliometria", "análise", "bibliométrica/o", "gestão", "revisão", "avaliação" e "produção" e estão fortemente relacionadas ao delineamento dos estudos investigados e à área macro do evento ciências administrativas, contábeis e afins.

Além disso, com base na nuvem de palavras exposta, destaca-se a frequência de palavras como "inovação", "estratégia", "políticas", "públicas", "governança", "empreendedorismo", "desempenho" e "comportamento", as quais podem indicar campos de pesquisa com maior incidência relacionada a esses estudos bibliométricos. As temáticas dos eventos com maior publicação de estudos bibliométicos, conforme sugere a Tabela 2, corroboram essa conclusão, sendo esses, Ensino e Pesquisa em Administração e Contabilidade (EPQ), Estratégia em Organizações (ESO), Gestão de Pessoas e Relações de Trabalho (GPR), Gestão de Ciência, Tecnologia e Inovação (GCT) e Gestão de Operações e Logística (GOL).

\section{Considerações finais}

Esta pesquisa teve por objetivo investigar a produção de estudos bibliométricos na área de ciências da administração, contábeis e afins em âmbito nacional. Para tanto, por meio da análise de 141 estudos publicados nos anais da AnPAD entre os anos de 2003 e 2019, foi possível verificar os principais aspectos que contemplam a evolução desses estudos. Os resultados evidenciaram que, embora tenha ocorrido um crescimento no número de estudos bibliométricos, este não foi linear, pois ao longo do período investigado, houve uma oscilação no número das publicações. Esse resultado não é inusitado, uma vez que se observou achados semelhantes no estudo de Teixeira, Iwamoto e Medeiros (2013).

Em relação ao evento que teve o maior número de estudos bibliométricos publicados, destaca-se o EnANPAD como líder no ranking, com as divisões temáticas do EPQ e ESO, resultado semelhantes ao de Teixeira, Iwamoto e Medeiros (2013). Quanto a fonte de buscas, apresentaram-se como as mais utilizadas a Web of Science, a AnPAD e a Scopus. Em estudo realizado por Cassundé, Barbosa e Mendonça (2018), esse resultado foi equivalente. No que 
tange a autoria e filiação institucional dos pesquisadores, percebeu-se uma associação entre esses aspectos, uma vez que o autor está vinculado a uma ou mais instituição. Diante desse contexto, observou-se que os artigos são escritos na maioria das vezes por 2 ou 3 autores e o número máximo de publicação relativa a estudo bibliométrico por autor foi 6 . As três instituições que podem ser consideradas como referência, pela incidência de publicaç̃es desse tipo de estudo, foram a UNINOVE, USP e UNB.

Relativa a abrangência dos estudos, houve um equilíbrio no número de publicações que utilizou os estratos nacional e internacional, denotando que ainda há espaço para colaborações internacionais na área objetivo de análise desse estudo, como forma de tornar as pesquisas ainda mais produtivas. No que diz respeito ao uso de softwares de apoio à análise de dados dos estudos bibliométricos, os resultados indicaram que $60,29 \%$ dos trabalhos fizeram uso de alguma ferramenta, sendo Microsoft ${ }^{\circledR}$ Office Excel, SPSS, UCINET $\circledast$ e BibE$x c e l$ as mais utilizadas. Segundo Ruas e Pereira (2014) essas ferramentas computacionais permitem explorar o processo de recuperação de informação das bases de dados do começo ao fim, facilitando a análise dos dados por parte do pesquisador. Com relação da frequência de palavras-chave, as terminologias de maior ocorrência foram "bibliometria", "análise", "bibliométrica/o", "gestão", "revisão", "avaliação" e "produção". Pode-se dizer que as mesmas estão fortemente relacionadas ao delineamento dos estudos investigados e à área macro do evento ciências administrativas, contábeis e afins. A Figura 4 ilustra a síntese das principais evidências desse estudo.

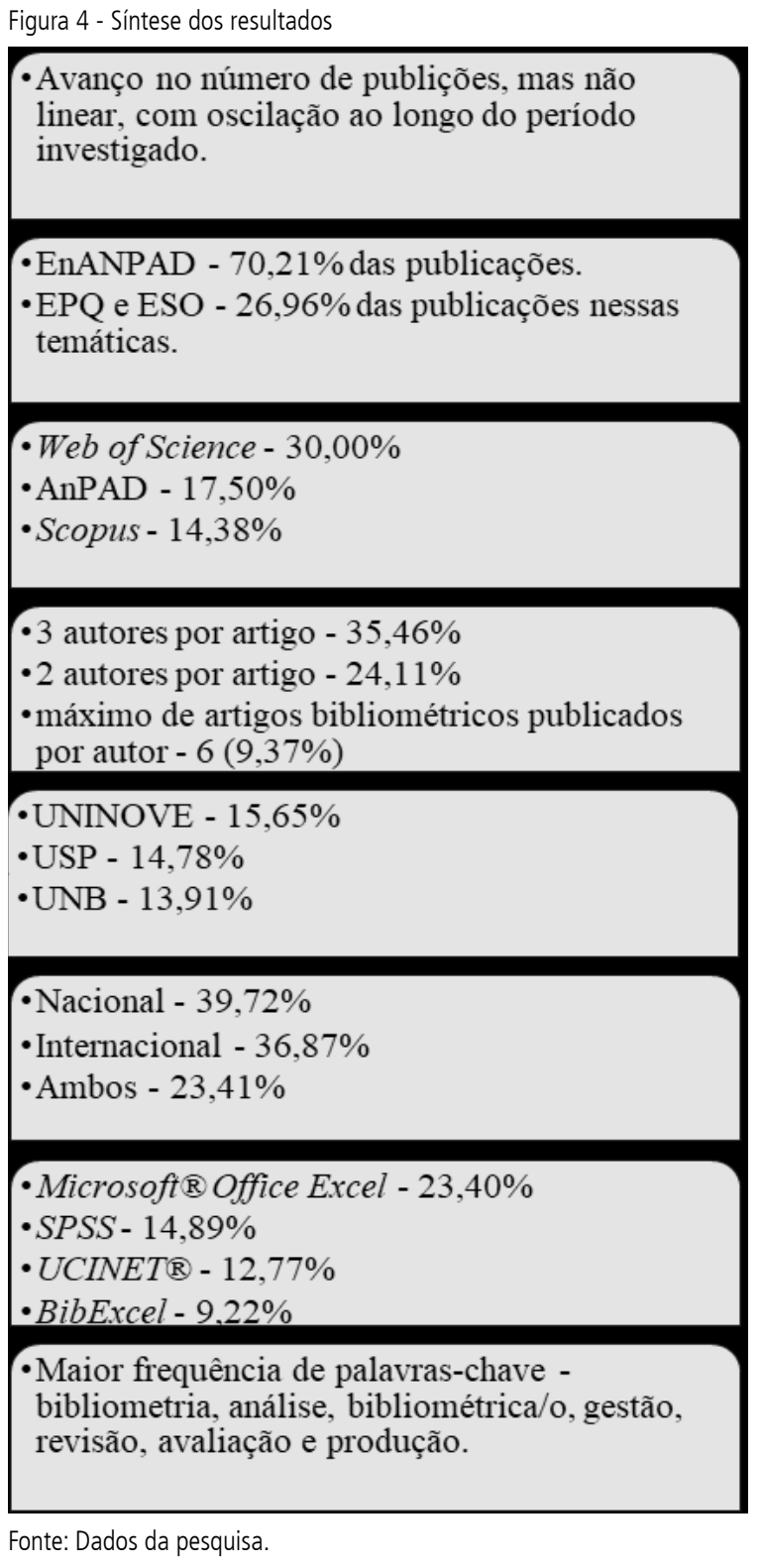

Diante desses resultados, essa pesquisa possibilitou uma visão ampla da produção acadêmica nos principais eventos da área no Brasil, como também mostrou o comportamento desses estudos ao longo dos anos de publicação, criando um cenário e um norte aos leitores, pesquisadores, e demais interessados no assunto, podendo assim, promover o avanço científico nesse campo de conhecimento. Sendo assim, a partir deste estudo bibliométrico, observou-se que existem oportunidades para o desenvolvimento de novas pesquisas, como por exemplo trabalhos que apresentem comparações entre estudos de revisão sistemática de literatura, incluindo os estudos bibliométricos.

Ainda, sugere-se pesquisas futuras que contemplem um maior escopo de análise, como por exemplo, periódicos nacionais e internacionais, que dis- 
cutam a aplicação das leis da bibliometria, bem como análises de redes de colaboração entre os pesquisadores. Essas sugestões serviriam para suprir as limitações dessa pesquisa e ainda a principal considerada como a análise somente de artigos publicados nos eventos da AnPAD, fato que delimita as conclusões da pesquisa apenas ao contexto nacional.

\section{Referências bibliográficas}

ALVARADO, R. U. A Bibliometria no Brasil. Revista Ciência da Informação, Brasilia, p 91-105, Jul./Dez. 1984. ARAÚJO, R. M. et al. Periódicos em ação: um estudo exploratório-bibliométrico na área de Administração, Ciências Contábeis e Turismo, Perspectivas em Ciência da Informação, v.19, n.1, p.90-114, jan./mar. 2014.

ALBUQUERQUE, C. A. de; ANDRADE, A. S. C. G. de; JOIA L. A. Estudos de Caso em Administração da Informação: uma Década de História a partir dos Anais do EnANPAD. Anais do EnANPAD: Rio de Janeiro, 2010.

BOTELHO, L. L. R. et al. Revisão Bibliométrica sobre Mudança Organizacional e Aprendizagem Gerencial em uma Organização Intensiva em Conhecimento. Anais do EnANPAD: Rio de Janeiro, 2011.

CAFÉ, L. M. A.; BRÄSCHER, M. Organização da informação e bibliometria. Encontros Bibli: revista eletrônica de biblioteconomia e ciência da informação, v. 13, n. 1, p. 54-75, 2008.

CASSUNDÉ, F. R. de S. A.; BARBOSA, M. A. C.; MENDONÇA, J. R. C. Entre revisōes sistemáticas e bibliometrias: como tem sido mapeada a produçãa acadêmica em Administração no Brasil?. Informação \&

CHIARETO, J. et al. Avaliação Estratégica de Desempenho em Organizaçōes de Saúde: Uma Bibliometria de dos últimos 25 anos. Anais do 3Es: Brasilia, 2015

FONSECA, E. N. Bibliografia estatística e bibliometria: uma reivindicação de prioridades. Ciência da Informação, Brasilia, v. 2, n.1, p. 5-7, 1973

FONSECA, P. H.; MATITZ, Q. R. S. Diversidade Humana em Estudos de Administração e Estratégia: Um Estudo Bibliométrico de Produção Qualificada de 2014 A 2017. Anais do EnANPAD. Curitiba, PR. 2018.

FREITAS, B. A. de; SILVA, D. A. da; PRADO, T. A. R. Análise da produção científica sobre contabilidade pública no Âmbito brasileiro: um estudo aplicando os conceitos das leis bibliométricas Lotka e Bradford nos principais periódicos da área de Administração e Contabilidade. Contabilidade, gestão e agronegócio

GUEDES, V. L.; BORSCHIVER, S. Bibliometria: Uma ferramenta estatística para a Gestão da Informação e do Conhecimento, em Sistemas de Informação, de Comunicação e de Avaliação Científica e Tecnológica. Encontro Nacional de Ciência da Informação, jun. 2005.

HEINZMANN, L. M.; JÚNIOR, V. M. V. Assédio Moral: Análise da (quase) Maioridade de um Tema por meio da Bibliometria. Anais do EnEO. Belo Horizonte, MG. 2016.

HOPPEN, N.; MEIRELLES, F. S. Sistemas de Informação: Um panorama da pesquisa cientifica entre 1990 e 2003. RAE, v. 45, n.1, p.24-35, 2005

JONKERS, K. DERRICK, G. E. The Bibliometric Bandwagon: Characteristics of Bibliometric Articles Outside the Field Literature. Journal of The American Society for Information Science and Technology, v.63, n.4, p.829-836, 2012

LAWANI, S. M. Bibliometrics: its theoretical foundations, methods and applications. Libri, v. 31, n. 1, p. 294-315, 1981.

MENEGHINI, R.; PACKER, A. L. The extent of multidisciplinary authorship of articles on scientometrics and bibliometrics in Brazil. Interciência, v.35, n.7, p.510-514. 2010.

NASCIMENTO, R. L. et al. Poder na Administração, Administração Pública, Contabilidade e Turismo. Revista pensamento contemporâneo em administração, v. 11, n. 5, p. 153-166. 2017. OLIVEIRA, A. S. F. Governança da educação básica no Brasil: da análise bibliométrica ao instrumento de medida.
Anais do EnAPG. Salvador, BA. 2019 .

Sh REVISTA SOCIAIS \& HUMANAS - VOL. 32 / № 3 - 2019
OLIVEIRA, A. B. B. et al. Análise Bibliométrica da Produção Acadêmica Sobre Políticas Públicas na Bioeconomia e suas Potencialidades. Anais do EnAPG. Salvador, BA. 2019

OTLET, P. Traité de documentation; le livre sur le livre; théorie et pratique. Bruxelles, Editiones Mundaneum, 1934

PINTO, L. S. F.; MORI, S. S. O.; JUNIOR, C. D S. Inovação na contramão: uma bibliometria dos artigos relativos aos usuários que inventam. Anais do EnANPAD. Costa do Sauípe, BA. 2016.

PORTAL CAPES. Missão e Objetivos. Disponível em: <https://www-periodicos-capes-gov-br.ez47.periodicos.capes, gov.br/index.php?option=com_pcontent\&view=pcontent\&alias =missao-objetivos\&ltemid=109>. Acesso em: 01/07/2019.

PRITCHARD, A. Statistical bibliography or bibliometrics? Journal of Documentation, v. 25, n. 4, p. 348-349, Dec 1969.

ROIG-TIERNO, N.; GONZALEZ-CRUZ, T. F.; LLOPIS-MARTINEZ, J. An overview of qualitative comparative analysis: A bibliometric analysis. Journal of Innovation \& Knowledge, v. 2, n. 1, p. 15-23, 2017

RUAS, T. L.; PEREIRA, L. Como construir indicadores de Ciência, Tecnologia e Inovação utilizando Web of Science, Derwent World Patent Index, Bibexcel e Pajek?. Perspectivas em Ciência da Informação, v. 19, n. 3, p. $52-81,201$

SANTOS, R. N. M. dos; KOBASHI, N. Y. Bibliometria, Cientometria, Informetria: Conceitos e Aplicaçōes. Tendências da Pesquisa Brasileira em Ciência da Informação, Brasilia, v.2, n.1, p.155-172, Jan./Dez. 2009

SENGUPTA, I. N. Bibliometrics, informetrics, scientometrics and librametrics: an overview. Libri, v. 42, n. 2, p. 99-135, 1992.

SORDI, J. O. de et al. A Abordagem Design Science no Brasil Segundo as Publicações em Administração da Informaçăo. Anais do EnANPAD: Rio de Janeiro, 2013

SPLITTER, K.; da ROSA, C. A.; BORBA, J. A. Uma Análise das Características dos Trabalhos "Ditos" Bibliométricos Publicados no Enanpad entre 2000 e 2011. Anais do EnANPAD: Rio de Janeiro, 2012.

TEIXEIRA, M. L. M.; IWAMOTO, H. M.; MEDEIROS, A. L. Bibliometric studies (?) In Administration: discussing the transposition of purpose. Administração-Ensino e Pesquisa, v. 14, n. 3, p. 423-452, 2013.

THANUSKODI, S. Journal of Social Sciences: A Bibliometric Study, Journal of Social Sciences, 2010, v. 24:2, p. 77 80, DOI: 10.1080/09718923.2010.11892847.

REINTA, F. T. et al. Metodologia de pesquisa bibliográfica com a utilização de método multicritério de apoio decisão. Prod., São Paulo, v. 24, n. 3. 2014

WORD CLOUDS. Disponível em: <https://www.wordclouds.com/>. Acesso em: 01/07/2019.

VANTI, N. A. P. Da bibliometria à webometria: uma exploração conceitual dos mecanismos utilizados para medir o registro da informação e a difusão do conhecimento. Ciência da informação, v. 31, n. 2, p. 152-162, 